\title{
Uso excesivo del celular, calidad de sueńo y soledad en jóvenes de la ciudad de Arequipa
}

\author{
Excessive Use of Cell Phone, Quality of Sleep and Loneliness \\ in Young People in The City of Arequipa \\ Atena Sota Velásquez \\ Universidad Católica San Pablo, Arequipa, Perú \\ (iD https://orcid.org/oooo-0oo2-0322-6967 \\ Melani Maldonado López \\ Universidad Católica San Pablo, Arequipa, Perú \\ (iD) https://orcid.org/oooo-oooz-2934-3395 \\ Correspondencia: melani.maldonado@ucsp.edu.pe \\ Manuel Ytuza Cusirramos \\ Universidad Católica San Pablo, Arequipa, Perú \\ (iD https://orcid.org/oooo-0oo2-3304-1453 \\ María del Carmen Cornejo Torres \\ Universidad Católica San Pablo, Arequipa, Perú \\ (iD) https://orcid.org/oooo-0oo3-1906-9959 \\ Yessenia Cusirramos Carpio \\ Universidad Católica San Pablo, Arequipa, Perú \\ iD https://orcid.org/oooo-0003-0817-338X
}

\section{Resumen}

Se dice que el uso excesivo de celulares entre adolescentes y jóvenes puede causar efectos negativos en su salud psicológica y física, así como en su calidad de sueño. En diversas investigaciones se menciona que las personas que informan un mayor nivel de soledad también refieren menos sueño. Sin embargo, todavía es necesario profundizar, la relación entre el uso excesivo al celular, la calidad del sueño y la soledad, ya que no existe suficiente evidencia para definirla. Se seleccionó una muestra de 397 estudiantes universitarios de la ciudad de Arequipa, utilizando como criterio de inclusión que cursen 
estudios en una universidad y que se encuentren entre el rango de edad de 17 a 25 años. Los estudiantes fueron invitados a completar un cuestionario anónimo que contenían datos demográficos, la Escala de Soledad de la Universidad de California los Ángeles ULS-8, el Îndice de Calidad de Sueño de Pittsburgh ICSP y el cuestionario autoadministrable del Uso Problemático del Celular para adolescentes. Se encontró que las variables soledad, calidad del sueño y uso problemático del celular tienen una relación significativa entre sí. Los resultados obtenidos corroboran la hipótesis planteada, encontrando que, sí existe relación entre las variables soledad, calidad del sueño y uso problemático del celular en estudiantes universitarios.

Palabras clave: Uso del celular, calidad de sueño, soledad, jóvenes.

\section{Resumen}

It has been said that the excessive use of cell phones among adolescents and young people can have negative effects on their psychological and physical health, as well as their quality of sleep. In various investigations it is mentioned that people who report a higher level of loneliness also report less sleep. However, it is still necessary to deepen our understanding of the relationship between excessive cell phone use, sleep quality and loneliness, as there is limited evidence to support a full description of this association. A simple of 397 university students from the City of Arequipa was selected, using as inclusion criteria attendance at a university and being between the age range of 17 to 25 years. The students were invited to complete an anonymous questionnaire containing demographic questions, the University of California Los Angeles ULS-8 Loneliness Scale, the Pittsburgh ICSP Sleep Quality Index, and the self-administered Questionnaire on Problematic Use of Cell Phones by Teenagers. It was found that the variables loneliness, sleep quality and problematic cell phone use have a significant relationship with each other. The results obtained corroborate the proposed hypothesis, finding that there is a relationship between the variables of loneliness sleep quality and problematic use of the cell phones in university students. Keywords: Cell phones use, sleep quality, loneliness, young people. 


\section{Introducción}

Desde la aparición de los celulares inteligentes en 1997, capaces de enviar mensajes de texto, acceder a internet, redes sociales, música, videos, chats, correos electrónicos y todo tipo de aplicaciones que el usuario desee descargar; se dio un aumento descontrolado en el uso de esta tecnología, suscitando inquietudes entre los investigadores no solamente por su potencial de crear adicción sino por su capacidad de fortalecer o de debilitar los vínculos entre las personas, según el uso que se le dé al dispositivo (Peñuela et al., 2014).

En los últimos años el Instituto Nacional de Estadística e Informática (INEI) informó que durante el trimestre julio-agosto-setiembre de 2017, en el 9o.6 $\%$ de los hogares de Perú existe al menos un miembro que tiene celular y que del total de población que usa Internet, el $30.5 \%$ accedió a Internet únicamente por celular. Entre los que accedieron en mayor proporción a Internet se tiene a la población joven de 19 a 24 años de edad con $79.4 \%$ y el grupo de 12 a 18 años de edad con $72 \%$. A partir de estos datos se refleja que, en el Perú, en su mayoría, adolescentes y jóvenes tienen un celular y lo utilizan no sólo para llamar o mandar mensajes, sino que también se conectan a Internet (INEI, 2017).

Actualmente en varias investigaciones realizadas en Asia y Europa (Menglong \& Liya, 2017; Pamuk \& Atli, 2016; Peper \& Harvey, 2018) se evidencia que la nueva tecnología dio inicio a un marcado deterioro psicológico y social de los usuarios que en su mayoría son jóvenes, adolescentes y púberes. Hardell, Carlberg, Söderqvist, Hansson y Mild (2008) realizaron una encuesta sistemática, descubriendo que los adolescentes involucrados en el uso excesivo de teléfonos móviles reportan tener más problemas de salud, incluyendo fatiga, depresión, dolor de cabeza, ansiedad, trastorno de la atención y trastornos del sueño.

En investigaciones sobre exposiciones a campos electromagnéticos de radiofrecuencia, asociadas a síntomas de enfermedad, se ha reportado en algunos usuarios de celulares efectos en su salud durantey después de su uso, como mareos, dolores de cabeza, sensaciones de ardor, hormigueo en la piel de la cabeza y extremidades, perturbaciones del sueño, cansancio, pérdida de capacidad atencional, disminución del tiempo para reaccionar, pérdida de retención, problemas en el sistema digestivo, taquicardia y malestar general, entre otros. Estos síntomas coinciden con los del estrés y no deberían separarse de sus efectos nocivos (Roösli, 2008). Loughran et al. (2005) encontraron que la exposición a largo plazo a dispositivos electrónicos tales como los celulares reducen la frecuencia de los movimientos oculares durante el período de sueño de movimientos oculares rápidos, acorta la latencia del sueño y eleva la potencia del electroencefalograma (EEG) en el sueño temprano de 11,5-12,25 Hz.

En el Perú, el neurólogo y director del Instituto Peruano de Neurociencias, el doctor David Lira mencionó que los 
trastornos de sueño consisten en una patología de la sociedad moderna, que en la actualidad tiene una estrecha relación con el uso prolongado de dispositivos electrónicos. En los países en los que se ha medido la relación entre el uso de dispositivos electrónicos y la falta de sueño se ha determinado que los que lo usan antes de dormir tienen más dificultades para conciliar el sueño. El doctor Lira comentó que este problema se está presentando cada vez más entre los jóvenes, debido a la permisividad de los padres con respecto al empleo de tabletas y celulares hasta altas horas de la noche. Así, el Dr. Lira menciona que:

Presentan un sueño fraccionado básicamente por ansiedad. Como están conectados toda la noche no duermen de forma profunda. Están a la expectativa de que les contesten los mensajes. Su sueño es superficial. Están pendientes de cualquier ruido para levantarse a contestar. Eso impacta en su desempeño académico (Lira, citado en Garay, 2016)

En la actualidad, el $84 \%$ de los adolescentes duerme con su celular en la cama (Gaglianone, 2015) y se ha visto repercusiones en su calidad de sueño. La calidad del sueño es un fenómeno complejo que resulta difícil de definir y medir de forma objetiva. Ésta incluye aspectos cuantitativos del sueño, tales como la duración del sueño, latencia del sueño, números de despertares y aspectos puramente subjetivos como "profundidad" o "descanso" del sueño (Buysse et al., 1989). La calidad de sueño se refiere al hecho de dormir bien durante la noche y tener un buen funcionamiento durante el día (Domínguez et al., 2007; Rosales et al., 2007) y no solamente es importante como factor determinante de la salud, sino como elemento propiciador de una buena calidad de vida (Rodríguez et al., 2002; Sierra, 2006).

El sueño es una necesidad fisiológica básica para la supervivencia de la persona. A esto se da, la importancia de conseguir la cantidad apropiada de las fases REM (Rapid Eye Movement) - NREM (No Rapid Eye Movement) y el tiempo total que pueda dormir un individuo. Además, el bienestar psicológico se puede comprobar en el funcionamiento mental (Guevara \& Torres, 2016). En una investigación realizada por Dexter, Bijwadia, Schilling y Applebaugh (2003), plantearon que la participación de los estudiantes en las actividades familiares, sociales y deportivas, así como los horarios de trabajo de los padres pueden producir conflictos a la hora de dormir. La angustia y el aumento de la confianza en las relaciones entre pares, así como la disponibilidad de medios de comunicación como es el celular, tienden a retardar las horas de sueño. La televisión, los videojuegos y las computadoras presentan el mismo efecto.

Diversos hallazgos sugieren que sujetos que consiguen dormir bien y tienen una buena calidad de sueño, poseen mayor capacidad de concentración, autocontrol y realización de tareas personales y profesionales, ya que dormir poco tiempo altera negativamente la capacidad de concentración, el aprendizaje y la memoria del individuo. Una mala calidad de sueño es 
un síntoma que con frecuencia aparece asociado a la fatiga, la disminución de las capacidades cognitivas y motoras durante el día y una mayor incidencia de accidentes de tránsito y laborales (Blasco et al., 2002). Aunque se ha demostrado en distintas investigaciones que el uso excesivo y la adicción al celular afectan la calidad del sueño de los adolescentes y jóvenes, existe poca evidencia que describa los mecanismos involucrados. Mrunal y Jaimala (2015) sugirieron que la adicción al celular entre los adolescentes correlaciona positivamente y de modo significativo con su soledad.

El sentimiento de soledad es un elemento inherente a la condición humana, es probable que todas las personas experimentan este sentimiento durante el curso de su vida, en forma transitoria o duradera (Carvajal-Carrascal \& Caro-Castillo, 2009; Rotenberg \& Hymel, 1999). Se presenta en todas las culturas, y ha sido identificado en diversas publicaciones recientes en diversos países como Australia, Canadá, Estados Unidos y Perú (Cuny, 2001). Existen definiciones que describen la soledad como un estado donde la persona se encuentra sola, sin compañía alguna simplemente, pero en realidad la soledad es un estado de ánimo que hace que las personas se sientan vacías e indeseadas. Sin embargo, para Cacioppo y Cacioppo (2016) es una elección personal, un lugar donde nace la creatividad, la concentración y el sentido de la vida. Weiss (2008) afirma que los sentimientos de soledad son considerados como una deficiencia en los sistemas de interacción interpersonal. Él diferenció entre soledad de tipo emocional y soledad de tipo social. La soledad emocional aparece en ausencia de apego emocional y ocurre como resultado de la pérdida de intimidad con personas cercanas como padres, pareja o hijos. Por el contrario, la soledad social aparece en ausencia de una red social de apoyo, por ejemplo, como consecuencia de la pérdida de amigos, vecinos y colegas. La soledad de tipo social puede propiciar hacia la aparición de soledad emocional.

Asher y Paquette (2003) afirman que, aunque la soledad está influenciada por características objetivas de las relaciones sociales (frecuencia del contacto social y número de amigos), está más influenciada por características subjetivas como la satisfacción con las relaciones sociales y la aceptación social percibida. Según Mrunal y Jaimala (2015), la soledad es una emoción experimentada por un individuo que desea un nivel de contacto diferente en la que se encuentra actualmente, es decir, la soledad es la angustia que resulta de las discrepancias entre las relaciones sociales ideales y las percibidas. Esta discrepancia cognitiva se ve reflejada en los pensamientos, sentimientos y comportamientos, los cuales repercuten en la salud y el bienestar de la persona.

En una investigación sobre la relación entre Internet y la soledad realizada por Kraut etal. (1998), se encontró que, aunque el uso de la red era fundamentalmente comunicativo, existía una disminución en la comunicación familiar, en los círculos sociales y un incremento de la depresión y la soledad. También descubrieron que, a mayor uso de Internet, mayores eran 
sus efectos. La soledad como experiencia desagradable se produce cuando la red social de una persona es deficiente de forma significativa en calidad o cantidad, y se asocia con sentimientos negativos (Borges et al., 2008).

Según Kersting (2016) parte de la cuestión de la soledad es que la mayoría de las personas que utilizan las redes sociales no sólo publican, sino que pasan mucho tiempo mirando los post de todos los demás, dónde están, a dónde van y qué están haciendo. La exposición constante a las experiencias de vida "perfectas" de todos los demás provoca sentimientos de soledad, depresión y ansiedad. Las personas que se han involucrado significativamente con sus celulares pueden estar expuestas a una disminución en el tiempo asignado a otras relaciones sociales, especialmente las relaciones cara a cara, de interacción, que puede provocar que se sientan solas. El investigador Arslan (2013), encontró que el uso problemático del celular asciende a medida que aumenta el tiempo de conversación, sin embargo, el aumento del tiempo que uno habla disminuye el nivel de soledad en los adolescentes.

Algunos investigadores (Doane \& Thurston, 2014; Hutchison \& Gerstein, 2016) consultaron a adolescentes con respecto a su estrés diario, emociones, soledad y calidad del sueño, y encontraron que una mayor presión conduce a un menor tiempo de sueño y que la soledad es una importante variable de control entre el estrés y la duración del sueño. En otras investigaciones se menciona que las personas que informan un mayor nivel de soledad también refieren menos sueño. A partir de los estudios mencionados se ha revelado la existencia de correlaciones negativas entre la adicción a los celulares de los jóvenes y su calidad de sueño, así como la correlación positiva entre la soledad y la adicción al celular (Oguz \& Cakir, 2014). Es por este motivo que consideramos importante investigar si existe relación entre la soledad, el uso excesivo de los celulares y la calidad de sueño en estudiantes universitarios, ya que existen pocas evidencias al respecto.

Por lo tanto, el objetivo de la investigación es analizar la relación entre el uso excesivo del celular, la soledad y la calidad de sueño en una muestra de jóvenes universitarios de la ciudad de Arequipa.

\section{Método}

\section{Diseño de la investigación}

La investigación corresponde a una estrategia asociativa con un diseño comparativo transversal, ya que en este estudio se analiza la relación entre las variables uso problemático del celular, calidad de sueño y soledad (Ato et al., 2013).

\section{Muestra}

Se trabajó con una muestra de 397 estudiantes universitarios, de tres universidades de la ciudad de Arequipa: Universidad Católica San Pablo, Universidad Nacional de San Agustín y Universidad Alas Peruanas. Las edades de los estudiantes fluctúan entre los 17 
y 25 años y la muestra está conformada por 149 varones (37.5\%) y 248 mujeres $(62.5 \%)$. Se cumplieron con los siguientes criterios de inclusión: que los participantes fueran tanto varones y mujeres en condición de estudiantes universitarios, cuyas edades estuvieran comprendidas entre los 17 y 25 años, y que hablaran español como lengua materna (Tabla 1).

Tabla 1.

Datos demográficos de los estudiantes universitarios

\begin{tabular}{|lccc|}
\hline \multicolumn{1}{|c}{ Variables } & Sujetos & Cantidad & Porcentaje \\
\hline Sexo & Varones & 149 & $37.5 \%$ \\
& Mujeres & 248 & $62.5 \%$ \\
\hline Hijo Único & Sí & 70 & $17.6 \%$ \\
& No & 327 & $82.4 \%$ \\
\hline Número & Ninguno & 70 & $17.6 \%$ \\
de hermanos & 1 & 155 & $39.0 \%$ \\
& 2 & 99 & $24.9 \%$ \\
& $3-5$ & 68 & $17.1 \%$ \\
& Más de 5 & 5 & $1.3 \%$ \\
\hline $\begin{array}{l}\text { Condición } \\
\text { económica }\end{array}$ & Pobre & 7 & $1.8 \%$ \\
familiar & Relativamente pobre & 13 & $3.3 \%$ \\
& Mediano & 347 & $87.4 \%$ \\
& Relativamente rico & 30 & $7.6 \%$ \\
\hline
\end{tabular}

\section{Instrumentos}

Para medir la variable soledad, se utilizó la Escala de Soledad para Adultos de la UCLA (Versión 3). La versión inicial de esta escala fue desarrollada por Russell, Peplau y Fergusson (1980), que en estudios con muestras de estudiantes reportaron buena consistencia interna $(\alpha=.96)$, y confiabilidad obtenida con el método test-retest fue de .73 , en un periodo de tiempo de dos meses. La validez concurrente indicó relación de los puntajes de las escalas con otros indicadores de soledad, relaciones sociales y estados afectivos (Russell, Peplau \& Fergusson, 1980). Adicionalmente, y con respecto a validez de criterio, se ha demostrado que la UCLA Loneliness Scale es un buen criterio para probar diferencias en la experiencia subjetiva de soledad emocional y social (Róquez \& Martínez, 2015). Posteriormente, Russell, Peplau y Fergusson (1980), reportaron los datos de validez, confiabilidad y dimensionalidad de la escala para la versión 3. Esta 
versión se ha desarrollado para tratar de corregir algunas limitaciones de la escala que pueden alterar su confiabilidad. A través de la literatura se reporta amplia utilización en muestras de adolescentes y adultos, y buenas cualidades psicométricas (Russell, Peplau \& Fergusson, 1980). Esta escala consta de 20 ítems, los cuales están redactados de la siguiente manera: 11 de forma positiva (no hay sentimiento de soledad) y 9 de manera negativa (hay sentimiento de soledad). Para responderla, a los participantes se les pide que indiquen el grado de soledad en una escala tipo Likert de 1 al 4. Para calificar las puntuaciones se suman juntas y las puntuaciones altas indican altos niveles de soledad.

Para medir la calidad de sueño se utilizó el Índice Pittsburg de Calidad de Sueño (PSQI) validado en una muestra peruana, la cual consta de nueve preguntas; las cuatro primeras son de respuesta subjetiva mientras que las otras 5 se responden en una escala de tipo Likert: (o) "Ninguna vez en el último mes", (1) "Menos de una vez a la semana", (2) "Una o dos veces a la semana y (3) "Tres o más veces a la semana”. Así mismo el ICSP está compuesta por siete componentes: calidad de sueño subjetiva, latencia del sueño, duración del dormir, eficacia del sueño habitual, alteraciones del sueño, uso de medicamentos para dormir y disfunción diurna; que al sumar el valor de cada componente da la calificación global del ICSP (Luna-Solís et al., 2015). Cabe resaltar que en cuanto a la calificación, a mayor puntaje indica una muy mala calidad de sueño y a menor puntaje indicará una mejor calidad de sueño.

También se utilizó la Escala de Uso Problemático del Celular, la cual fue traducida y adaptada por Manuel Ytuza, se eligió como juez a la Mg. Paula Delgado Cuzzi, docente de la Universidad Católica San Pablo, para que validara la traducción y la adaptación. La escala fue recuperada del artículo "Development of a problematic mobile phone use scale for University students: Validity and reliability study" (Pamuk \& Atli, 2016). Dicha escala cuenta con cinco subescalas que son: privación, resultados adversos, problema del control e interacción-evitación.

Por último, fue utilizado un cuestionario para reunir la información general de los sujetos tales como sexo, condición de hijo único, condición económica familiar, número de hermanos y tiempo que los padres pasan en casa por año. Los datos se muestran en la Tabla 1.

\section{Procedimiento}

La evaluación se realizó dentro de las instalaciones de las respectivas universidades, a las cuales se accedió por medio de permisos consentidos por los profesores. La recolección de datos se realizó en diversos horarios de estudio, durante los turnos mañana, tardey noche. Durante la encuesta, se proporcionó a los participantes información sobre la metodología y el propósito del estudio. Los cuestionarios se distribuyeron en estricta conformidad con el principio de consentimiento 
informado. Una vez hecho esto, procedieron a completar los instrumentos en presencia de los investigadores para que pudieran absolver cualquier interrogante que tuvieran.

\section{Criterios para el análisis de datos}

Se utilizó el programa estadístico SPSS versión 22.o para realizar el procesamiento de los datos. Para revisar la distribución de los datos se realizó un análisis de la asimetría y curtosis, teniendo como resultado, en su mayoría, los datos tienen una distribución no normal. Por lo que se eligió la prueba de correlación de Spearman, relacionando las variables de calidad de sueño, soledad y uso problemático del celular. En cuanto al establecimientodepercentiles en la escala del uso problemático del celular se hicieron cortes en los percentiles 25, 50 y 75 (Lloret-Segura et al., 2014); para diferenciar a los jóvenes universitarios que tenían una percepción de soledad de los que no, se analizó la media. Y para diferenciar la buena calidad de sueño de la mala calidad de sueño se realizó un análisis de la puntuación Z de cada ICSP, teniendo como referencia el intervalo [-2; 2] (Lloret-Segura et al., 2014).

\section{Resultados}

\section{Estadística descriptiva}

En la Escala de Soledad UCLA (versión 3), se hizo un análisis de la mediana, donde el $47.9 \%(n=190)$ de estudiantes universitarios no perciben soledad mientras que el $52.1 \%(\mathrm{n}=207)$ sí perciben soledad. En la Escala de Uso problemático del Celular, se encontró que 103 universitarios obtuvieron una puntuación baja (28.9\%), 103 un puntaje alto (28.9\%) y 191 tienen un uso moderado (48.2 \%). Estos resultados nos indican que las personas con bajo puntaje no tienen un uso problemático del celular, mientras que las personas con un puntaje alto sí tienen un uso problemático del celular y los restantes tienen un uso moderado. En los resultados del Índice de Calidad de Sueño de Pittsburgh, se encontró que 43 universitarios reportan tener una muy buena calidad de sueño (10.8 \%), 177 tienen una buena calidad de sueño (44.6\%), 154 tienen una mala calidad de sueño (38.8 \%) y 23 una muy mala calidad de sueño (5.8\%).

\section{Análisis de correlación}

La Tabla 2 muestra que las variables soledad y calidad del sueño correlacionan positivamente y de manera significativa, es decir que a más puntaje en el ICSP mayor será la percepción de soledad. Cabe recordar que a mayor puntaje en el ICSP se indica una mala o muy mala calidad de sueño. La soledad presenta una correlación significativa con el uso problemático del celular, es decir que a mayor uso problemático del celular mayor será la percepción de soledad, así como a mayor percepción de la soledad mayor será el uso problemático del celular. Así mismo, el uso problemático del celular y la calidad de sueño están significativamente correlacionadas, es decir que más uso del celular la calidad de sueño será de menor calidad. Lo cual valida la hipótesis de investigación; ya que hay respaldo estadístico acerca de las relaciones entre dichas variables. 
Tabla 2.

Correlación entre las variables

\begin{tabular}{|lccc|}
\hline & Soledad & $\begin{array}{c}\text { Calidad } \\
\text { de sueño }\end{array}$ & $\begin{array}{c}\text { Uso problemático } \\
\text { del celular }\end{array}$ \\
\hline Soledad & 1 & & \\
\hline Calidad de sueño & $.161^{* *}$ & 1 & \\
\hline Uso problemático del celular & $.143^{* *}$ & $.182^{* *}$ & 1 \\
\hline
\end{tabular}

${ }^{* *} \mathbf{p}<.01$

\section{Discusión}

En nuestra sociedad tan cambiante y globalizada, las tecnologías como el celular ocupan un espacio importante en la vida de las personas, impactando en sus estilos de vida, sus actitudes, conductas, hábitos, etc. (Castellana et al., 2007). El uso desmedido de este dispositivo ha generado distintas investigaciones y variadas consideraciones por estudiosos, debido a que su uso que inicialmente es placentero puede llegar a generar dependencia psicológica (Castellana et al., 2007) y repercutir en actividades que anteriormente eran gratificantes como el dormir o salir con amigos. Visto de esta forma, el uso problemático o excesivo del celular consiste en el fracaso de resistir el impulso de realizar llamadas, enviar mensajes o entrar a las redes sociales; que puede a lo largo, tener consecuencias negativas en la vida de la persona.

Llegar a comprender qué factores se asocian a este uso desmedido es adentrarnos a revisar diversos temas de índole bio-psico-social. Dentro de la literatura diversas investigaciones apuntan que estar constantemente pendientes de mensajes entrantes o de si el celular suena o vibra, es más un comportamiento asociado a sentimientos de soledad. Según un estudio realizado por Peper y Harvey (2018), se encontró que a pesar de que los teléfonos inteligentes y las posibilidades de conectarnos a Internet en cualquier lugar y momento, nos acercan a personas que tenemos lejos geográficamente o a quienes no podemos ver tan seguido; con el tiempo puede ser un comportamiento excesivo $o$ adictivo que aumenta el riesgo de depresión, ansiedad y los sentimientos de soledad. En otro estudio publicado en el American Journal of Preventive Medicine, se encontró que el uso intensivo de plataformas como Facebook, Snapchat e Instagram se asocian con sentimientos de aislamiento social entre los adultos jóvenes (Primack et al., 2017).

En una investigación publicada en el Journal of Social and Clinical Psychology, Hunt, Marx, Lipson y Young (2018) reportaron que pasar mucho tiempo en redes sociales como Facebook, Instagram y Snapchat aumenta la sensación de soledad y genera depresión. En nuestro estudio, 
por ejemplo, evidenciamos resultados similares ya que la soledad presenta una correlación significativa con el uso problemático del celular lo que nos indica que a mayor uso de este dispositivo móvil mayor será el incremento del sentimiento de soledad. Asimismo, dentro de nuestros resultados se refleja que el uso excesivo del celular y la calidad de sueño están significativamente correlacionadas, es decir que, a más uso del celular, la calidad de sueño será menor.

Diversos estudios respaldan que el uso de estos dispositivos móviles, especialmente cerca de las horas de ir a dormir, está asociado con una peor calidad del sueño. Christensen et al. (2016) encontraron en su investigación que el uso de los celulares particularmente en las horas en las que los participantes dicen que van a la cama, estuvo asociado con un mayor tiempo para quedarse dormido y peor calidad de sueño durante la noche. Otro estudio realizado por Chang, Aeschbacha, Duffya y Czeislera (2014) muestra que la luz azul emitida por los teléfonos inteligentes (y otros aparatos digitales) pueden suprimir la función de melatonina del cuerpo, una hormona que induceal cansancioy contribuye a la sincronización de los ciclos de sueño y vigilia. Según investigadores del Hospital Brigham y de Mujeres de Boston, leer o escribir en celulares y tabletas en la cama momentos antes de dormir, retrasa la sensación de sueño y afecta la calidad del descanso durante toda la noche (citado en diario El Salvador, 2015).

Otra investigación hecha por un equipo de la escuela médica de Harvard comparó los efectos de leer en un dispositivo móvil o uno de papel, donde encontraron que las personas que utilizan libros electrónicos o apps de lectura con luz integrada o retroiluminados tardan más en dormir, lo cual deriva en una peor calidad del sueño durante la noche y en un mayor cansancio por la mañana (BBC citado en diario El Salvador, 2015). La OMS (2017) por su parte, señaló que existen efectos a corto plazo como consecuencia entre la interacción de la energía radioeléctrica y el cuerpo. Uno de ellos es el calentamiento de los tejidos, lo que afecta la función cognitiva, el sueño, el ritmo cardíaco e incluso genera ansiedad y estrés.

Por último, el impacto del móvil en la calidad del sueño es especialmente importante entre los más jóvenes de la casa. De hecho, un estudio realizado a unos 1000 estudiantes australianos entre 13 y 16 años afirma que los dispositivos móviles no solo influyen en la privación del sueño, sino que también puede afectar la salud mental de éstos, derivando en bajos estados de ánimo o periodos de depresión. Por otro lado, es importante no excluir la posibilidad de que algunas personas no pueden dormir por una razón completamente diferente, y como no pueden hacerlo, usan su celular sólo para pasar el tiempo.

Con respecto a la relación entre calidad de sueño y soledad, diversos autores afirman que existen factores ambientales que pueden desatar el insomnio, algunos de ellos son: el ruido, la falta de ventilación y los viajes. No obstante, el problema podría tener un origen físico o psicológico, como el estrés y la soledad. Un estudio 
realizado por Matthews et al. (2017) se encontró que la reducción en la calidad del sueño es una de las muchas formas en las que la soledad afecta a las personas. Los resultados asociaron los sentimientos de soledad con una peor calidad general del sueño. Sin embargo, esto debe analizarse con mayor profundidad en estudios posteriores, debido a los diferentes factores asociados en la baja calidad de sueño.

En un estudio realizado por Yan, Liu y Lei (2015) se investigó la adicción al celular, la calidad del sueño y la soledad en 346 estudiantes. Los resultados muestran que una alta prevalencia a la adicción al celular provoca mayor sensación de soledad y peor calidad del sueño. En nuestro estudio los resultados indican que las variables soledad, calidad de sueño y uso excesivo del celular correlacionan positivamente de manera significativa; es decir que los jóvenes universitarios que tienen un mayor uso del celular y menor calidad de sueño presentan tener mayor soledad. De esta manera respondemos a la pregunta inicial encontrando relación entre estas variables. Podemos concluir diciendo que el uso excesivo del celular es un problema psicológico, social y físico; que merece ser investigado en nuestro país con mayor contundencia.

Por otra parte, es necesario involucrar en posteriores investigaciones otras variables que también afectan el uso excesivo del celular y la calidad de sueño. Además, con estudios más detallados en un futuro cercano, se podría lograr mejores interpretaciones y explicaciones sobre la relación entre estas variables en jóvenes y adolescentes. Finalmente, consideramos de gran importancia y necesidad que se realicen estudios sobre la soledad en jóvenes y adolescentes que utilizan dispositivos móviles y celulares inteligentes en el Perú.

\section{Financiamiento}

El estudio fue autofinanciado.

\section{Conflicto de intereses}

Los autores declaran no tener ningún conflicto de interés. 


\section{Referencias}

Asher, S., \& Paquete, J. (2003). Loneliness and peer relations in childhood. Current Directions in Psychological Sciences, 12(1), 75-80. https://doi.org/10.111/1467-8721.01233

Arslan, A., \& Ünal, A.T. (2013). Examination of cell phone usage habits and purposes of education faculty students. International Journal of Human Sciences, 10(1), 182-201. https://www.j-humansciences.com/ojs/index.php/IJHS/article/view/2523

Ato, M., López, J. J., \& Benavente, A. (2013). Un sistema de clasificación de los diseños de investigación en psicología. Anales de psicología, 29(3), 1039-1055. https://doi. org/10.6018/analesps.29.3.178511

Blasco, J.E., Llor E.B., García, M., Saenz, M., \& Sánchez, M. (2002). Relación entre la calidad del sueño, el burnout y el bienestar psicológico en profesionales de la seguridad ciudadana. Mapfre Medicina, 13(1), 259-267. https://sid.usal.es/idocs/ f8/art8655/relacion.pdf

Borges, Á., Prieto, P., Ricchetti, G., Hernández-Jorge, C., \& Rodríguez, E. (2008). Validación cruzada de la factorización del Test UCLA de Soledad. Psicothema, 2o(4), 924-927. https://www.redalyc.org/pdf/727/72720462.pdf

Buysse, D. J., Reynolds, C. F., Monk, T. H., Berman, S. R., \& Kupfer, D. J. (1989). The Pittsburgh sleep quality index: A new instrument for psychiatric practice and research. Psychiatry Research, 28(19), 193-213. https://doi.org/10.1016/0165-1781(89)90047-4

Cacioppo, J., \& Cacioppo, S. (2016). La soledad, una nueva epidemia. Diario EL PAÍS. https://elpais.com/elpais/2016/o4/o6/ciencia/1459949778_182740.html

Castellana, M., Sánchez, X., Graner, C., \& Beranuy, M. (2007). El adolescente ante las Tecnologías de la Información y la Comunicación: Internet, móvil y videojuegos. Papeles del Psicólogo, 28(3), 196-204. https://www.redalyc.org/pdf/778/77828306.pdf

Carvajal-Carrascal, G., \& Caro-Castillo, C. (2009). Soledad en la adolescencia: Análisis del concepto. Aquichan, 9(3), 281-96. https://www.redalyc.org/pdf/741/741121470o8.pdf

Christensen, M. A., Bettencourt, L., Kaye., L., Sai, M., Nguyen, K., Olgin, L. E., Pletcher, M..., \& Marcus, G. M. (2016). Direct measurements of smartphone screen-time: Relationships with demographics and sleep. Plos ONE, 11(11). doi.org/10.1371/ journal.pone.0165331 
Cuny, J. A. (2001). Actitud y sentimiento de soledad en un grupo de adolescentes universitarios de Lima. Persona, 4(1), 111-128. https://revistas.ulima.edu.pe/index. $\mathrm{php} /$ Persona/article/view/816

Chang, A., Aeschbacha D., Duffya J., \& Czeislera C. (2014). Evening use of light-emitting eReaders negatively affects sleep, circadian timing, and next-morning alertness. Proceedings of the National Academy of Sciencies of the United Stades of America, 112(4), 1232-1237. www.pnas.org/cgi/doi/10.1073/pnas.1418490112

Dexter D., Bijwadia J., Schilling D., \& Applebaugh G. (2003) Sleep sleepiness and school start times: a preliminary study. World Medicine Journal, $102(1)$, 44-46. https://www.semanticscholar.org/paper/Sleep\%2Csleepiness-and-school-start-times\%3A-a-study.-Dexter-Bijwadia/ cae4a6981d992b107ad419b35e48a91c2653de6d?p2df

Doane, L. D., \& Thurston, E. C. (2014). Associations among sleep, daily experiences, and loneliness in adolescence: Evidence of moderating and bidirectional pathways. Journal of Adolescence, 37(2), 145-154. https://doi.org/10.1016/j.adolescence.2013.11.009

Domínguez, S., Oliva, M., \& Rivera, N. (2007). Prevalencia de deterioro del patrón del sueño en estudiantes de enfermería en Huelva. Revista Electrónica Semestral de Enfermería, 1(10), http://revistas.um.es/eglobal/article/viewFile/346/318

El Salvador (2016). ¿Por qué los celulares afectan la calidad del sueño? Diario El salvador. https://www.elsalvador.com/vida/144184/ por-que-los-celulares-afectan-la-calidad-del-sueno/

Garay, K. (2016). Smartphones: 41 \% de jóvenes peruanos lo considera imprescindible. Diario El Peruano. https://elperuano.pe/noticia-el-3o-de-poblacion-tiene-seriosproblemas-sueno-39164.aspx

Gaglianone, V. (2015). Adolescentes adictos al celular. Diario La Opinión. https:// laopinion.com/2015/o1/22/adolescentes-adictos-al-celular/

Guevara, K. G., \& Torres, E. T. (2016). Calidad de sueño y somnolencia diurna en conductores de taxi de una empresa privada en Lima Metropolitana-Santiago de Surco. (Tesis de Licenciatura). Universidad Peruana Cayetano Heredia, Lima, Perú. http://repositorio.upch.edu.pe/bitstream/handle/upch/647/Calidad\%2ode\%2o sue\%c3\%b1o\%2oy\%2osomnolencia\%2odiurna\%2oen\%2oconductores\%2ode\%2o taxi\%2ode\%2ouna\%2oempresa\%2oprivada\%2oen\%2oLima\%2oMetropolitana\%2o-\%2oSantiago\%2ode\%2oSurco\%2c\%202016.pdf?sequence=1\&isAllowed=y 
Hardell, L., Carlberg, M., Söderqvist, F., \& Hansson Mild, K. (2008). Meta-analysis of long-term mobile phone use and the association with brain tumours. International Journal of Oncology, 32(5), 1097-1103. http//:doi.org/10.3892/ijo.32.5.1097

Hunt, M. G., Marx, R., Lipson, C., \& Young, J. (2018). No more FOMO: Limiting social media decreases loneliness and depression. Journal of Social and Clinical Psychology, 37(10), 751-768. https://guilfordjournals.com/doi/pdfplus/10.1521/jscp.2018.37.10.751

Hutchison, A. N., \& Gerstein, L.H. (2016). The Impact of Gender and Intercultural Experiences on Emotion Recognition. Revista de Cercetare si Interventie Sociala, 54(1), 125-141. https://www.ceeol.com/search/article-detail?id=520443

Instituto Nacional de Estadística e Informática (INEI) (2017). El 30.5 \% accede a Internet desde su smartphones, según el INEI. Diario Gestión. https://gestion. pe/peru/30-5-accede-internet-smartphones-segun-inei-223558

Kersting, T. (2016). Disconnected. How to reconnect our digitally distracted kids. http:// tomkersting.com/

Kraut, R., Patterson, M., Lundmark, V., Kiesler, S., Mukophadhyay, T., \& Scherlis, W. (1998). Paradoja de Internet: ¿una tecnología social que reduce la participación social y el bienestar psicológico? Psicólogo estadounidense, 53(9), 1017-1031. https:// doi.org/10.1037/0003-066X.53.9.1017

Lloret-Segura, S., \& Ferreres-Traver, A., \& Hernández-Baeza, A., \& Tomás-Marco, I. (2014). El análisis factorial exploratorio de los ítems: una guía práctica, revisada y actualizada. Anales de Psicología, 30(3), 1151-1169. https://revistas.um.es/analesps/ article/view/analesps.30.3.199361

Loughran, S. P., Wood, A. W., Barton, J. M., Croft, R. J., Thompson, B., \& Stough, C. (2005). The effect of electromagnetic fields emitted by mobile phones on human sleep. Neuroreport, 16(17), 1973-1976. https://doi.org/10.1097/o1.wnr.0ooo186593.79705.3C

Luna.-Solís, Y., Robles-Arana, Y., \& Agüero-Palacios, Y. (2015). Validación del índice de calidad de sueño de Pittsburgh en una muestra peruana. Anles de la Salud Mental, 31(2), 23-30. https://www.academia.edu/34647099/VALIDACI\%C3\%93N_ DEL_\%C3\%8DNDICE_DE_CALIDAD_DE_SUE\%C3\%91O_DE_PITTSBURGH_EN_ UNA_MUESTRA_PERUANA_VALIDATION_OF_THE_PITTSBURGH_SLEEP_ QUALITY_INDEX_IN_A_PERUVIAN_SAMPLE 
Matthews, T., Danese, A., Gregory, A. M., Caspi, A., Moffitt, T. E., \& Arseneault, L. (2017). Sleeping with one eye open: loneliness and sleep quality in young adults. Psychological Medicine, 47(12), 2177-2186. https://doi.org/10.1017/Soo3329171700o629

Menglong, L. I., \& Liya, L. U. (2017). La influencia de la adicción al teléfono móvil en la calidad de sueño de estudiantes secundarios dejados atrás: el rol mediador de la soledad. Revista Argentina de Clínica Psicológica, 26(1), 71-81. https://www. redalyc.org/pdf/2819/281950399007.pdf

Mrunal B., \& Jaimala S. (2015). Mobile Phone Addiction and Loneliness among Teenagers. The International Journal of Indian Psychology, 2(1), 27-34. http://oaji. net/articles/2015/1170-1430026211.pdf

Organización Mundial de la Salud (OMS) (2017). Campos electromagnéticos y salud pública:teléfonos móviles. https://www.who.int/es/news-room/fact-sheets/detail/ electromagnetic-fields-and-public-health-mobile-phones

Oguz, E., \& Cakir, O. (2014). Relationship between the Levels of Loneliness and Internet Addiction. Anthropologist, 18(1), 183-189. https://doi.org/10.108o/o972 0073.2014.11891534

Pamuk, M., \& Atli, A. (2016). Development of a problematic mobile phone use scale for University Students: Valicity and Reliabity study. The Journal of Psychiatry and Neurological Sciences, 29(1), 49-59. https://dusunenadamdergisi.org/storage/ upload/pdfs/1585660689-en.pdf

Peper, E., \& Harvey, R. (2018). Digital Adicction: Increased loneliness, Anxiety and depression. Neuroregulation, 5(1), 3-8. https://doi.org/10.15540/nr.5.1.3

Peñuela, M., Patermina, J., Moreno, S., Camacho, L., Acosta, L., \& De León, L. (2014). El uso de los smartphones y las relaciones interpersonales de los jóvenes universitarios en la ciudad de Barranquilla (Colombia). Salud Uninorte, 30(3), 335-346. https://www.redalyc.org/articulo.oa?id=81737153008

Primack, B. A., Shensa, A., Sidani, J. E., Whaite, E. O., Lin, L., Rosen, D., ... \& Miller, E. (2017). Social media use and perceived social isolation among young adults in the US. American journal of preventive medicine, 53(1), 1-8. https://doi.org/10.1016/j. amepre.2017.01.010 
Rodríguez, R., Viegas, C., Abreu eSilva,A., \& Tavares, P. (2002). Daytimesleepinessandacademic performance in medical students. Arquivos de Neuropsiquiatria, 6o(1), 6-11. http:// www.scielo.br/scielo.php?script=sci_arttext\&pid=So004-282X2002000100002\&lng

Rosales, E., Egoavil, M., La Cruz, C., \& De Castro, J. (2007) Somnolencia y calidad del sueño en estudiantes de medicina de una universidad peruana. Anales de la Facultad de Medicina, 68 (2), 150-158. http://sisbib.unmsm.edu.pe/BVrevistas/ anales/v68n2/pdf/ao7v68n2.pdf

Röösli, M. (2008). Radiofrequency electromagnetic field exposure and non-specific symptoms of ill health: a systematic review. Environmental Research, 107(2), 277-287. https://doi.org/10.1016/j.envres.2008.02.003

Rotenberg, K. J., \& Hymel, S. (1999). Loneliness in childhood and adolescence. Cambridge University Press.

Róquez, E., \& Martínez, M. (2015). Propiedades psicométricas de la escala de soledad para adultos de la Universidad de California de los Ángeles. Versión 3. Universidad Rafael Urdaneta.

Russell, D., Peplau, L., \& Cutrona, C. (1980). The Revised UCLA Loneliness Scale. Concurrent and discriminant validity evidence. Journal of Personality and Social Psychology, 39(1), 472-48o. https://doi.org/10.1037/oo22-3514·39·3.472

Sierra, J. C. (2006). Calidad de sueño como factor relevante de la calidad de vida. En L. A. Oblitas (Ed.), Psicología de la Salud y Calidad de Vida (pp. 337-344). Thomson.

Weis, R. S. (2008). The experience of emotional and social isolation. MIT Press.

Yan, J., Liu, Q. S., \& Lei, C. H. (2015). Research on correlation among college students' mobile phone dependence, sleep quality and loneliness. Time Education, 19(1), 87-88.

Recibido: 3 de diciembre de 2020

Revisado: 23 de marzo de 2021

Aceptado: 30 de mayo de 2021 